\title{
Nuclear Factor $\kappa$ B-Dependent Neurite Remodeling Is Mediated by Notch Pathway
}

\author{
Sara Anna Bonini, ${ }^{1}$ Giulia Ferrari-Toninelli, ${ }^{1}$ Daniela Uberti, ${ }^{1}$ Mery Montinaro, ${ }^{1}$ Laura Buizza, ${ }^{1}$ Cristina Lanni, ${ }^{2}$ \\ Mariagrazia Grilli, ${ }^{3,4}$ and Maurizio Memo ${ }^{1}$ \\ ${ }^{1}$ Department of Biomedical Sciences and Biotechnologies, University of Brescia, 25123 Brescia, Italy, ${ }^{2}$ Department of Drug Sciences, Centre of Excellence in \\ Applied Biology, University of Pavia, 27100 Pavia, Italy, and ' 2 Laboratory of Neuroplasticity and Pain, Department of Chemical, Food, Pharmaceutical, and \\ Pharmacological Sciences, University of Piemonte Orientale “A. Avogadro,” and ${ }^{4}$ Drug and Food Biotechnology Center, 28100 Novara, Italy
}

In this study, we evaluated whether a cross talk between nuclear factor $\kappa \mathrm{B}(\mathrm{NF}-\kappa \mathrm{B})$ and Notch may take place and contribute to regulate cell morphology and/or neuronal network in primary cortical neurons. We found that lack of p50, either induced acutely by inhibiting $\mathrm{p} 50$ nuclear translocation or genetically in $\mathrm{p}^{-1-}$ mice, results in cortical neurons characterized by reduced neurite branching, loss of varicosities, and Notch1 signaling hyperactivation. The neuronal morphological effects found in $\mathrm{p}^{-1-}{ }^{-1-}$ cortical cells were reversed after treatment with the $\gamma$-secretase inhibitor DAPT ( $N$-[ $N$-(3,5-difluorophenacetyl)-1-alanyl 1]-S-phenylglycine $t$-butyl ester) or Notch RNA interference. Together, these data suggested that morphological abnormalities in $\mathrm{p}^{-1-}$ cortical neurons were dependent on Notch pathway hyperactivation, with Notch ligand Jagged 1 being a major player in mediating such effect. In this line, we demonstrated that the p50 subunit acts as transcriptional repressor of Jagged1. We also found altered distribution of Notch1 and Jagged1 immunoreactivity in the cortex of $\mathrm{p}^{-1-}$ mice compared with wild-type littermates at postnatal day 1 . These data suggest the relevance of future studies on the role of Notch/NF- $\kappa$ B cross talk in regulating cortex structural plasticity in physiological and pathological conditions.

\section{Introduction}

Functional and structural plasticity, often referred to as "neuroplasticity," is a fundamental brain property involving chemical, electrical, molecular, and cellular responses and leading to connection reorganization within and/or between brain regions. Potentially, a better understanding of the molecular events participating in neuroplasticity may provide relevant information for innovative therapeutic approaches in a variety of neurological disorders, including Alzheimer's disease, depression, autism, and schizophrenia, which have been associated with deregulated neuroplasticity (van Spronsen and Hoogenraad, 2010).

Among others, two important players involved in neuroplasticity regulation are nuclear factor $\kappa \mathrm{B}(\mathrm{NF}-\kappa \mathrm{B})$ and Notch signaling pathways. NF- $\kappa \mathrm{B}$ proteins are ubiquitously expressed transcription factors that play different roles depending on the cellular context in which they act and the dimer subunit composition. Their functional roles include regulation of nerve cell survival and plasticity, immune and inflammatory responses, proliferation, neurogenesis, apoptosis, angiogenesis, and oncogenesis (Grilli and Memo, 1999; Mattson and Meffert, 2006; Kaltschmidt and

\footnotetext{
Received March 3, 2011; revised May 30, 2011; accepted June 1, 2011.

Author contributions: M. Memo designed research; S.A.B., G.F.-T., D.U., M. Montinaro, L.B., and C.L. performed research; S.A.B. and M.G. analyzed data; S.A.B. wrote the paper.

This work was supported by grants from the Italian Ministry of Education, University and Research (PRIN 2007) and Regione Lombardia (Network-Enabled Drug Design). We thank Dr. Giovanna Cenini for critical discussions and comments on this manuscript, and Dr. Annamaria Lanzillotta and Dr. Valeria Bortolotto for technical support.

Correspondence should be addressed to Prof. Maurizio Memo, Department of Biomedical Sciences and Biotechnologies, University of Brescia, Viale Europa 11, 25123 Brescia, Italy. E-mail: memo@med.unibs.it.

DOI:10.1523/JNEUROSCI.1113-11.2011

Copyright $\odot 2011$ the authors $\quad 0270-6474 / 11 / 3111697-09 \$ 15.00 / 0$
}

Kaltschmidt, 2009). NF- $\kappa$ B-dependent transcriptional activity becomes relevant in neurons when axons and dendrites grow and synapses are formed, and remains high under basal conditions in most regions of the adult brain (Mattson, 2005). NF- $\kappa$ B signaling is also known to promote neurite outgrowth and to enhance the size and complexity of neuronal processes in the developing nervous system and in cultured neurons (Gutierrez et al., 2005; Gavaldà et al., 2009; Gutierrez and Davies, 2011). As for many other complex biological processes, the NF- $\kappa \mathrm{B}$ signaling pathway is likely to work in concert with others to regulate nerve cell survival and plasticity.

Notch is recognized as a key player in neurodevelopment (Artavanis-Tsakonas et al.,1990; Williams et al., 1995; de la Pompa et al., 1997), whereas in adulthood it appears to be involved in synaptic plasticity regulation and in "morphological maturation" of terminally differentiated neurons (Berezovska et al., 1999; Redmond et al., 2000; Wang et al., 2004). It has been previously shown that Notch activation induces neurite remodeling in different experimental models of neuronal cells by acting on cytoskeletal structures and modulates the expression of genes whose products are responsible for contact-dependent inhibition of dendrite outgrowth (Šestan et al., 1999; Ferrari-Toninelli et al., 2008, 2009).

Together, both Notch and NF- $\kappa \mathrm{B}$ signalings may potentially contribute by a reciprocal cross talk interaction to regulate neuronal functional and structural plasticity in physiological and pathological conditions. Numerous cellular contexts have been described wherein interaction of Notch and NF- $\kappa$ B signaling pathways takes place (Bash et al., 1999; Wang et al., 2001; Nickoloff et al., 2002; Espinosa et al., 2003; Shin et al., 2006; Osipo et 
al., 2008; Cao et al., 2010), but clear indications of their functional cross talk in cortical neurons and its potential relevance in CNS disorders remain to be demonstrated (Ang and Tergaonkar, 2007).

In this study, we evaluated whether a cross talk between NF- $\kappa \mathrm{B}$ and Notch may take place and contribute to regulate cell morphology and/or neuronal network in primary cortical neurons. The results obtained from in vitro and in vivo experiments support the idea that modulation of Notch/NF- $\kappa$ B cross talk may represent a potential target for pharmacological treatment of deregulated neuroplasticity.

\section{Materials and Methods}

Primary cortical neurons cultures. NF- $\kappa \mathrm{B}$ p50 ${ }^{-1-}$ (B6;129P2-Nfkb 1 tm 1 $\mathrm{Bal} / \mathrm{J}$; The Jackson Laboratory) and wild-type (wt) mice (B6;129PF2; The Jackson Laboratory) were maintained in high-efficiency particulate airfiltered THOREN units (THOREN Caging Systems) at the University of Piemonte Orientale animal facility, were kept three to four per cage, and had ad libitum access to food and water (Denis-Donini et al., 2008). Animal treatments were performed in accordance with the National Institutes of Health guidelines and approved by the local institutional animal care and use committee. To obtain primary cortical neurons, embryonic day 15 (E15) cortices were isolated from $\mathrm{p}^{-1-}$ and wt mice, pooled, mechanically dissociated into a single-cell suspension in Neurobasal Medium (Invitrogen) containing 2\% B27 supplement (N-B27 medium; Invitrogen), $500 \mu \mathrm{M}$ glutamine, $100 \mathrm{U} / \mathrm{ml}$ penicillin, and 100 $\mu \mathrm{g} / \mathrm{ml}$ streptomycin (Sigma-Aldrich), and centrifuged for $5 \mathrm{~min}$ at $200 \times$ g. Cells were plated onto poly-D-lysine (Sigma-Aldrich)-coated glass coverslips or dishes $\left(5 \times 10^{4} \mathrm{cells} / \mathrm{cm}^{2}\right)$ and cultured in N-B27 medium for $7 \mathrm{~d}$ in vitro (DIV). We assessed cell viability using the trypan blue exclusion assay, which provides a measure of cell membrane integrity. At 7 DIV, the medium was removed from the dishes and the attached cells were washed three times with PBS (Sigma-Aldrich). Cells were harvested with trypsin-EDTA (Invitrogen) and resuspended in medium. The cells were mixed 1:1 with 20\% trypan blue and counted using a hemocytometer. The number of blue (dead) cells was calculated and subtracted to the total cell number. Four separate experiments were performed from four different cell preparations.

Immunofluorescence and confocal analysis. Cells were plated at a density of $5 \times 10^{4}$ cells $/ \mathrm{cm}^{2}$ in a 24-well plate, grown on glass coverslip (coated with poly-D-lysine; Sigma-Aldrich), and then fixed. Cells were incubated in PBS (Sigma-Aldrich) containing $1 \%$ bovine serum albumin (BSA) (Sigma-Aldrich) and $0.2 \%$ Triton X-100 overnight at $4^{\circ} \mathrm{C}$ with the appropriate antibody. After rinses, cells were incubated with the secondary antibody in PBS for $1 \mathrm{~h}$ at room temperature. Slice were mounted using the Dako Fluorescent Mounting Medium and examined by Zeiss LSM 510 META confocal laser-scanning microscope (Carl Zeiss). Threedimensional images were performed with the LSM Image Browser software on $z$-stack scansion images ( $1 \mu \mathrm{m}$ interval sections) taken from fixed cells. For in vivo analysis, postnatal day 1 (P1) mice of either sex were killed, and brains were fixed by immersion in $4 \%$ buffered formalin. After overnight postfixation, brains were cryopreserved by dehydration in $30 \%$ sucrose solution, mounted in embedding medium, and cut in $15-\mu \mathrm{m}$-thick serial coronal brain sections using the cryostat. Brain sections were then incubated with primary antibody in PBS solution containing $3 \% \mathrm{BSA}$ and $0.3 \%$ Triton $\mathrm{X}-100$ at $4^{\circ} \mathrm{C}$ overnight. After rinses, brain sections were incubated with Alexa Fluor-conjugated (Invitrogen) secondary antibody in PBS/BSA (1\%) for $1 \mathrm{~h}$ at room temperature. Slice were mounted and examined by confocal microscopy.

Branching study. $\beta$ III-Tubulin-labeled neurons were visualized and digitally acquired using a Zeiss LSM 510 META confocal laser-scanning microscope (Carl Zeiss). To quantify the extent and complexity of neuronal processes, the Sholl profile analysis was undertaken (Sholl, 1953). Briefly, a series of concentric rings with regular radial increments $(20$ $\mu \mathrm{m})$ centered in the neuronal soma were traced, bifurcation (Bi) and terminal $(\mathrm{Ti})$ points of processes were counted in each ring and the number $(\mathrm{Xi})$ of processes intersecting each ring was calculated using the iterative equation $\mathrm{Xi}=\mathrm{Xi}-1+\mathrm{Bi}-\mathrm{Ti}$ (Gutierrez and Davies, 2007).
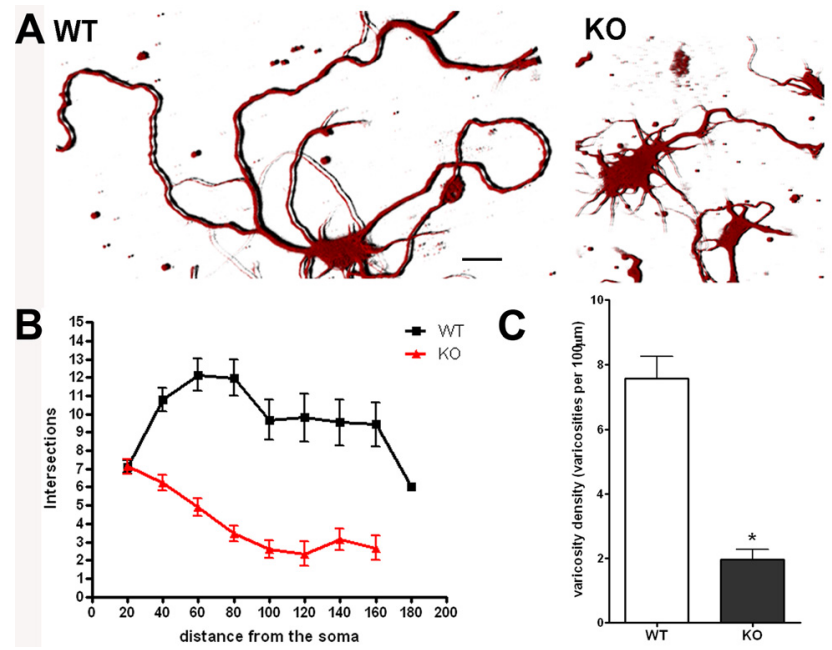

Figure 1. Neurite branching and varicosities are reduced in $\mathrm{p}^{-1-}$ cortical neurons. Reduced complexity and varicosity density of neuronal processes in $\mathrm{p}^{-0^{-1-}}$ (KO) compared with wild-type (WT) cortical cells. $A$, Representative 3 D images of cortical neurons from WT (left) and KO mice (right) stained with anti- $\beta$ III-Tubulin antibody. Scale bar, $20 \mu \mathrm{m}$. B, Graphic representation of branching quantification performed using the Sholl profile analysis. Data are expressed as mean \pm SEM. C, Quantification of varicosity loss in KO neurons compared with WT. Varicosity density was measured counting the number of varicosities in $100 \mu \mathrm{m}$ neurite length. Data are expressed as mean \pm SEM. ${ }^{*} p<0.0001$.

Antibodies. The following antibodies were used: monoclonal anti$\beta$ III-Tubulin (Promega) (working dilution: 1:1000 for immunofluorescence); monoclonal anti-GAPDH (Millipore) (working dilution: 1:1000 for immunoblotting); monoclonal anti-Notch1 [which recognizes both Notch1 full-length and Notch intracellular domain (NICD)] (SigmaAldrich) (working dilution: 1:100 for immunofluorescence and 1:1000 for immunoblotting); polyclonal anti-Jagged1 (Abcam) (working dilution: 1:500 for immunoblotting, 1:100 for immunofluorescence).

Conjugated CY3, CY2 (Jackson ImmunoResearch Laboratories), and FITCH (Sigma-Aldrich) were used as secondary antibodies.

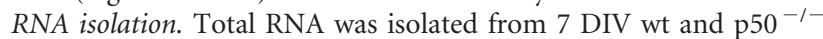
cortical cells $\left(\sim 2.5 \times 10^{6}\right.$ cells $)$ using the RNeasy kit (QIAGEN) and digested with the RNase-free DNase set (QIAGEN), according to the manufacturer's protocol. Quality of RNA samples was tested by RNA electrophoresis to ensure nucleic acid integrity.

Quantitative real-time PCR. One microgram of total RNA from wt and $\mathrm{p} 0^{-1-}$ cortical cells was transcribed into cDNA using murine leukemia virus reverse transcriptase (Promega) and oligo-dT(15-18) as a primer (final volume, $50 \mu \mathrm{l}$ ). Parallel reactions containing no reverse transcriptase were used as negative controls to confirm the removal of all genomic DNA. Murine-specific primers were designed using the Primer3 software (http://frodo.wi.mit.edu) (Rozen and Skaletsky, 2000). The oligonucleotide sequences of the primers (M-Medical) used are as follows: Notch1, forward primer, 5'-TGA GAC TGC CAA AGT GTT GC-3', reverse primer, 5'-GTG GGA GAC AGA GTG GGT GT-3'; Jagged1, forward primer, 5' -CAG TGC CTC TGT GAG ACC AA-3', reverse primer, 5' AGG GGT CAG AGA GAC AAG CA-3'; Hes1, forward primer, 5' -CCC ACC TCT CTC TTC TGA CG-3', reverse primer, 5' ${ }^{\prime}$-AGG CGC AAT CCA ATA TGA AC-3'; GAPDH, forward primer, 5'-AAC TTT GGC ATT GTG GAA GG-3', reverse primer, $5^{\prime}$-ACA CAT TGG GGG TAG GAA CA-3'. Amplification and detection were performed with the iCYCLER iQ Q-RT-PCR Detection System (Bio-Rad); the fluorescence signal was generated by SYBR Green I. Samples were run in triplicate in a 25 $\mu \mathrm{l}$ of reaction mix containing $12.5 \mu \mathrm{l}$ of $2 \times$ SYBR Green Master Mix (Bio-Rad), $12.5 \mathrm{pmol}$ of each forward and reverse primer, and $2 \mu \mathrm{l}$ of diluted cDNA. Each PCR experiment included serial dilutions of a positive control for construction of the calibration curve, a positive and a negative DNA sample, and water blanks. The PCR program was initiated by $10 \mathrm{~min}$ at $95^{\circ} \mathrm{C}$ followed by 40 cycles, each one of $15 \mathrm{~s}$ at $95^{\circ} \mathrm{C}$ and 1 min at $56-62^{\circ} \mathrm{C}$. A subsequent dissociation curve analysis verified the 

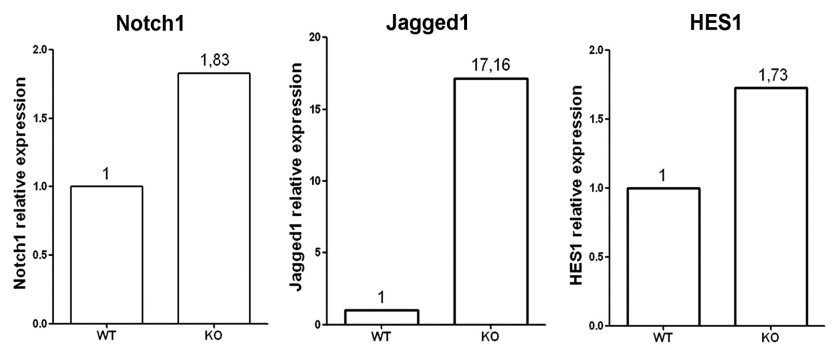

Figure 2. Notch1 pathway is upregulated in $\mathrm{p} 50^{-/-}$cortical cells. Q-RT-PCR was performed using RNA extracted from WT and K0 cortical cells to evaluate Notch1, Jagged1, and HES1 expression levels. Data are expressed as fold change of target gene expression in WT and $\mathrm{KO}$ cortical cells, normalized to the internal control gene (GAPDH). Data were analyzed according to the comparative $C t$ method.
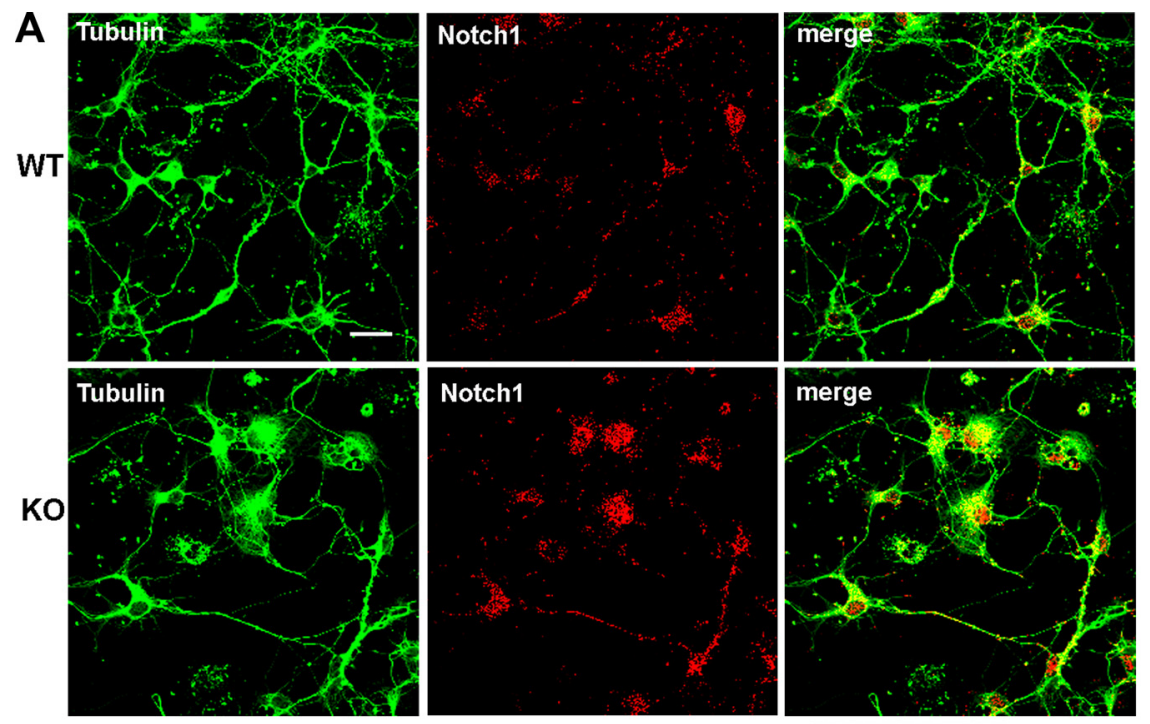

B

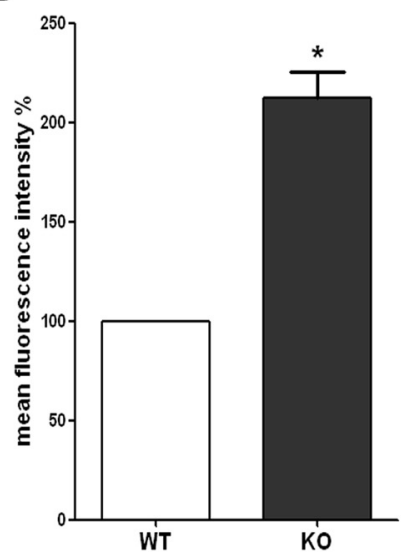

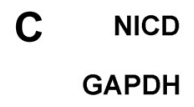

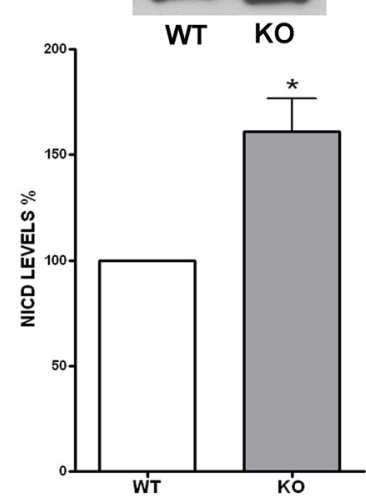

Figure 3. Notch 1 protein expression levels are increased in $\mathrm{p50}^{-1-}$ cortical cells. $\boldsymbol{A}$, Representative confocal images of cortical cells stained with anti- $\beta$ III-Tubulin (green) and anti-Notch1 (red) antibodies. Pictures show an increase of Notch1-positive staining in KO compared with WT cortical cells. Scale bar, $20 \mu \mathrm{m}$. B, Quantification of Notch1 immunostaining fluorescence intensity. Values are expressed as percentage of mean fluorescence intensity (\% mean \pm SEM) and are from at least 40 cellular fields. ${ }^{*} p<$ 0.001 versus WT. C, Representative immunoblot of WT and K0 cortical cell lysates using an anti-Notch1 antibody. Graph: Densitometric analysis of NICD levels measured by Western blot in WT and KO cortical cells. Data are normalized to the GAPDH signal, expressed as mean $\pm S E M$, and are obtained from three experiments run with two different cell preparations. ${ }^{*} p<0.01$.

products specificity. Gene expression levels are presented as fold change in target gene expression in the test ( ${\mathrm{p} 50^{-1-}}^{-}$cells) normalized to the internal control gene (GAPDH) and relative to the calibrator (wt cells). Results were estimated as $C t$ values; the $\Delta C t$ was calculated as the mean of the $C t$ for the target gene minus the mean of the $C t$ for the internal control gene. The $\Delta \Delta C t$ represented the mean difference between the $\Delta C t$ of the test minus the $\Delta C t$ of the calibrator. The $N$-fold differential expression in the target gene of the test compared with calibrator was expressed as $2^{-\Delta \Delta C t}$. Data analysis and graphics were performed using run in triplicate for each gene.

Western blots. Protein extracts were prepared from 7 DIV cortical cells from wt and $\mathrm{p} 50^{-/-}$embryos. Western blotting was performed using $6-12 \%$ SDS polyacrylamide gels with $20-50 \mu \mathrm{g}$ of protein extract loaded per lane. Nitrocellulose filters were incubated with prifollowed by HRP-conjugated secondary antibodies. Membranes were stripped and reprobed with anti-GAPDH antibody. Densitometric analysis was performed using Quantity One software system (BioRad), and each band was normalized to GAPDH signal in each lane and expressed as percentage.

Cell treatments. Ten micromolar $\gamma$-secretase inhibitor $N$-[N-(3,5-difluorophenacetyl)-1alanyl 1]-S-phenylglycine $t$-butyl ester (DAPT) (Calbiochem/EMD Biosciences) was added to the culture medium of 4 DIV cortical cells, and cells were analyzed after $72 \mathrm{~h}$. The DAPT treatment resulted in $34 \%$ reduction in Notch 1 protein levels and $48 \%$ reduction in NICD protein levels.

siRNA probes targeted to Notch1 receptor were purchased from Dharmacon. The mousespecific Notch1 interference was performed using an Accell SMARTpool siRNA mixture (DHE-041110-00) containing a mixture of four siRNAs targeting the Notch 1 gene (NM 008714). A nontargeting Accell siRNA pool (DHD-001910-10) was used as a control in all siRNA transfection experiments. Primary cortical neurons were transfected with Accell siRNAs in Neurobasal-B27 medium to preserve cell viability. Primary cortical neurons were treated with Accell siRNA probes after 4 DIV. Cells were cultured for 3 additional days after transfection with $1 \mu \mathrm{M}$ siRNA, according to the manufacturer's instructions, then treated, lysed, and subsequently analyzed for mRNA and protein contents. siRNA treatment effectiveness and specificity were validated by measuring Notch1 mRNA levels and using a scrambled siRNA (siRNA-). The siRNA treatment resulted in 58\% reduction in Notch 1 mRNA levels, $48 \%$ reduction in Notch 1 protein levels, and 30\% reduction in NICD protein, compared with vehicle-treated cells.

NF- $\kappa$ B SN-50 cell-permeable inhibitor peptide (Calbiochem/EMD Biosciences) was added to the culture medium at the concentration of 10 $\mu \mathrm{M}$ for $48 \mathrm{~h}$. To prove $\mathrm{SN}-50$ specificity, a mutant SN-50 (SN-50 mut) (Calbiochem/EMD Biosciences) was used at the concentration of 10 $\mu \mathrm{M}$ for $48 \mathrm{~h}$.

TNF- $\alpha$ (Invitrogen) was added to the culture medium at the concentration of $10 \mathrm{ng} / \mathrm{ml}$ for $24 \mathrm{~h}$.

Chromatin immunoprecipitation analysis. Chromatin immunoprecipitation (ChIP) analysis was performed essentially as described by Lanni et al. (2010). Protein complexes were cross-linked to DNA in living cells by adding formaldehyde directly to the cell culture medium at $1 \%$ final concentration. Chromatin extracts containing DNA fragments with an average size of $300 \mathrm{bp}$ were incubated overnight at $4^{\circ} \mathrm{C}$ with milk shaking using poly- 
clonal anti-p50 and anti-p65 antibodies (Santa Cruz). DNA-protein complexes were recovered with protein $\mathrm{A} / \mathrm{G}$-agarose (Santa Cruz). Before use, protein $\mathrm{A} / \mathrm{G}$ was blocked with $1 \mu \mathrm{g} / \mu \mathrm{l}$ sheared herring sperm DNA and $1 \mu \mathrm{g} / \mu \mathrm{l}$ BSA overnight at $4^{\circ} \mathrm{C}$, and then incubated with chromatin and antibodies for $3 \mathrm{~h}$ at $4^{\circ} \mathrm{C}$. PCR was performed using immunoprecipitated DNA and promoter-specific primers flanking the NF- $\kappa \mathrm{B}$ site for Jagged 1 (M-Medical) and specific primers for 36B4, used as housekeeping gene (kind gift from Dr. Delbarba, University of Milano, Milano, Italy). Primer sequences used were as follows: Jagged 1 promoter, forward primer, $5^{\prime}$-TTC AGG GGT GAT CAA GGA AG-3', reverse primer, $5^{\prime}$-TGG CAT ACT GGG AAT GTC AA3'; 36B4, forward primer, 5' -AGG ATA TGG GAT TCG GTC TCT TC- $3^{\prime}$, reverse primer, 5' TCA TCC TGC TTA AGT GAA CAA ACT-3'. Immunoprecipitation with nonspecific Igs (no $\mathrm{Ab}$ ) was performed as negative controls. PCR products were run on a $2.5 \%$ agarose gel and visualized with ethidium bromide staining using UV light. The amount of precipitated chromatin measured in each PCR was normalized with the amount of chromatin present in the input of each immunoprecipitation.

Statistical analysis. A $t$ test analysis or one-way ANOVA followed by Bonferroni's multiplecomparison test as post hoc analysis was performed. Data are expressed as mean \pm SEM of $n=3-5$ experiments. A value of $p<0.05$ was considered to be statistically significant.

\section{Results}

Neurite branching and varicosities are reduced in $\mathrm{p}^{50^{-/}}$cortical neurons Previous studies demonstrated that NF- $\kappa \mathrm{B}$ signaling is involved in regulation of neurite outgrowth, size, and complexity of cortical neuron arborizations (Gutierrez et al., 2005, 2008; Gavaldà et al., 2009). To gain insight into the role of NF- $\kappa \mathrm{B}$ in neuronal differentiation, we have taken advantage of the availability of mice carrying a homozygous deletion of the NF- $\kappa \mathrm{B} 1$ gene encoding the p50 subunit (Sha et al., 1995; Denis-Donini et al., 2008). Brain cortical neuroblasts were isolated from E15 wt and p50 ${ }^{-1-}$ embryos. Cells were grown on poly-D-lysine-coated glass coverslips and maintained in culture for $7 \mathrm{~d}$. Cells were then stained with an anti- $\beta$ IIITubulin antibody. Immunoreactivity and morphology were analyzed by confocal microscopy. We observed a remarkable difference in neurite branching of wt and $\mathrm{p}^{-1-}$ cultures. As shown in a representative image in Figure $1 A$, cortical cells from wt mice showed a complex and extended neuritic network, reminiscent of mature and differentiated cortical neurons. Conversely, the $\mathrm{p} 50^{-1-}$ derived cortical cells displayed short neurites and a poor branching phenotype (Fig. $1 A$, right panel). We then performed Sholl analysis, a valuable and widely used method for quantifying the extent and complexity of neuronal processes (Sholl, 1953; Gutierrez and Davies, 2007). An overall reduction in the spatial complexity of the neuritic arborizations was demonstrated in $\mathrm{p} 0^{-1-}$ derived cortical cells (Fig. $1 B$ ), with a significant decrease in neurite length and number of bifurcations when compared with wt derived cells.

The presence of varicosities is a well recognized morphological feature of differentiated cortical cells. They are regarded as presynaptic, dynamic structures that are able to remodel their morphology in response to a variety of stimuli (De Paola et al., 2003; Nikonenko et al., 2003; Udo et al., 2005; Umeda et al., 2005; Ferrari-Toninelli et al., 2009). Varicosities appear as membrane swellings of various size. They can represent more stable structures, giving origin to secondary branching neurites, filopodialike structures, or en passant varicosities, fast-turnover structures containing secretory granules (De Paola et al., 2003). We compared the presence of varicosities in wt and $\mathrm{p}^{-1-}$ cortical cells. Wt cortical cells in culture appeared to have long, thin, and varicose neurites, whereas $\mathrm{p} 50^{-I-}$ cortical cell neurites were short, thicker, and "smooth," since they were lacking varicosities. We quantified these differences by measuring the density of varicosities in $100 \mu \mathrm{m}$ neurite length. Varicosity densities were $7.57 \pm$

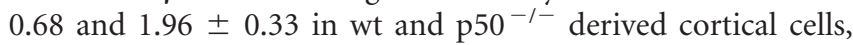
respectively (Fig. $1 C$ ).

Hyperactivation of Notch 1 pathway in absence of p50 subunit The morphological features observed in cortical neurons from p50-deficient mice were highly reminiscent of cortical cells in 
cultures after Notch pathway stimulation by a synthetic Jagged 1 ligand (Ferrari-Toninelli et al., 2008). We therefore investigated by quantitative real-time PCR (Q-RT-PCR) the expression levels of three major participants in the Notch signaling pathway, namely the Notch1 receptor, its ligand Jagged1, and the downstream bHLH family member HES1, a Notch1 target gene commonly used to detect Notch 1 pathway activation (Iso et al., 2003), in wt and $\mathrm{p}^{-1-}$ cortical cells. As reported in Figure 2, Notch1 and HES1 mRNA levels were increased by 83 and $73 \%$ in $\mathrm{p} 50^{-1-}$ compared with wt cultures. Remarkably, Jagged 1 mRNA levels increased by 17 -fold in $\mathrm{p}^{-1-}$ compared with wt cultures. Notch1 and Jagged1 protein expression was then evaluated by immunofluorescence. Cortical cells were double-stained with an anti-Notch 1 antibody, which recognizes both full-length Notch and its C-terminal intracellular fragment (NICD), and an anti$\beta$ III-Tubulin antibody. After immunostaining, we analyzed at least 40 fields (15-20 cells/field), derived from three different culture preparations. As shown in Figure $3 A$, Notch 1 immunoreactivity appeared to be increased in $\mathrm{p}^{-1-} 0^{-1}$ compared with wt cortical cells, considering both the number of Notch1-positive nuclei and the overall fluorescence intensity (Fig. 3A, merge). The total fluorescence intensity was quantified and expressed as percentage of mean fluorescence intensity. As reported in Figure $3 B$, fluorescence intensity was increased by twofold in $\mathrm{p}^{-1-} \mathrm{com}-$ pared with wt cultures. Western blot analysis using a specific anti-NICD antibody further supported the finding of an in-

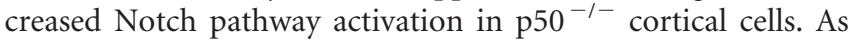
reported in Figure $3 C$, NICD protein levels were significantly increased (approximately $+60 \%$ ) in $\mathrm{p}^{-1-}$ compared with wt derived cortical cells.

Similarly, to evaluate Jagged 1 protein levels, wt and p50 ${ }^{-1-}$ cortical cells were double-stained with an anti-Jagged 1 and an anti- $\beta$ III-Tubulin antibodies (Fig. $4 A$ ). Fluorescence intensity of Jagged 1 immunostaining was quantified, and data were reported in Figure $4 B$. Fluorescence intensity was increased by $127 \%$ in p50 $0^{-1-}$ compared with wt cortical cells. Data were confirmed by Western blot analysis: the results from three different experiments demonstrated a significant increase $(+269 \%)$ in Jagged 1 level in $\mathrm{p} 0^{-1-}$ cultures (Fig. 4C).

Overall, these results demonstrate that Notch1 receptor, NICD, and Jagged 1 are significantly upregulated in the absence of p50 subunit.

Notch1 pathway inhibition restores neurite branching and varicosities in $\mathrm{p}^{50} \mathrm{O}^{-/-}$cortical cells

To investigate whether the observed morphological features of p50 $0^{-1-}$ cortical neurons were correlated to Notch1 pathway hyperactivation, the Notch1 pathway was inhibited either pharmacologically, using the $\gamma$-secretase inhibitor DAPT (Kanungo et al., 2008), or genetically, using the Notch1 siRNA approach (

Notch pathway inhibition by DAPT or siRNA NOTCH resulted in dramatic changes in $\mathrm{p} 0^{-1-}$ cortical neuron morphology. In particular, as shown by representative pictures in Figure 5A, 3-d-long treatment with DAPT or siRNA NOTCH (but not vehicle or scrambled siRNA) resulted in increased neurite branching and varicosities in $\mathrm{p} 0^{-1-}$ cells that became indistinguishable from wt cortical neurons. Varicosity density and branching were quantified in DAPT/siRNA NOTCH-treated $\mathrm{p} 50^{-1-}$ cells and compared with those of $\mathrm{p}^{-1-}$ vehicle or scrambled siRNA-treated cells. Quantitative data confirmed increased varicosity density (Fig. $5 B$ ) and branching (Fig. 5C) in DAPT and siRNA NOTCH-treated

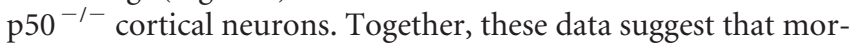

A
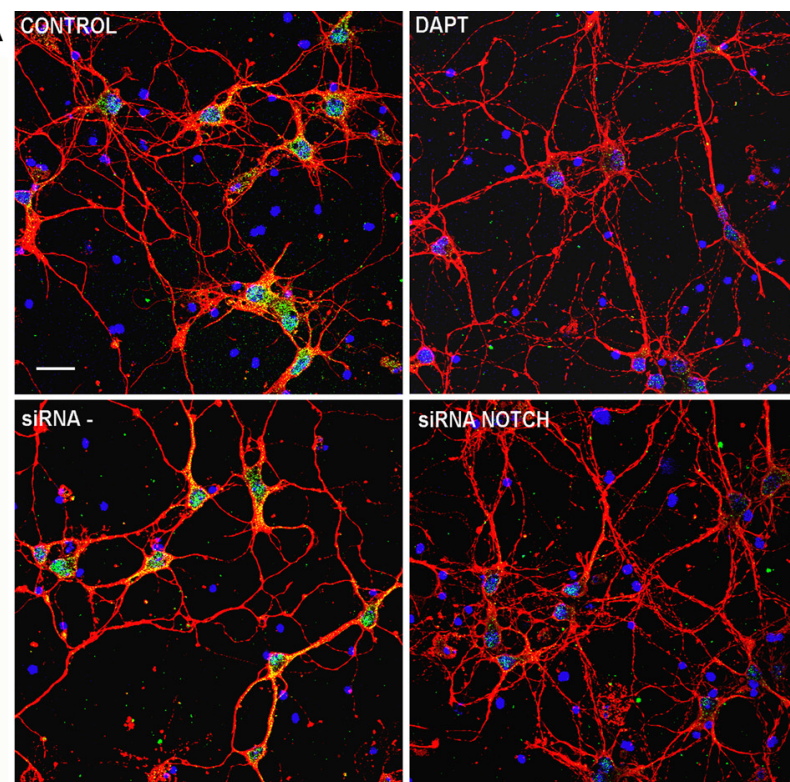

SIRNA NOTCH

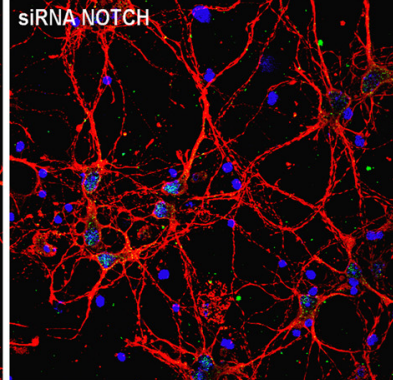

B

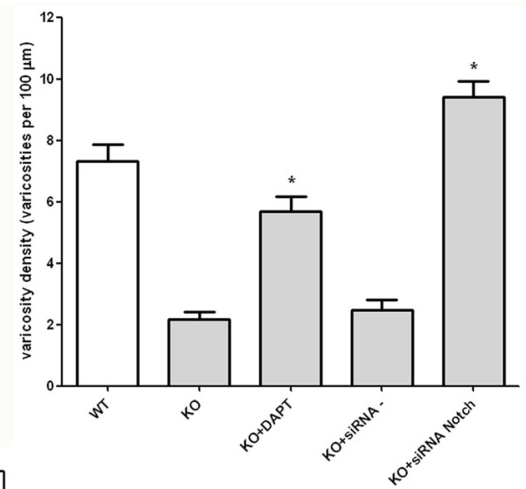

C

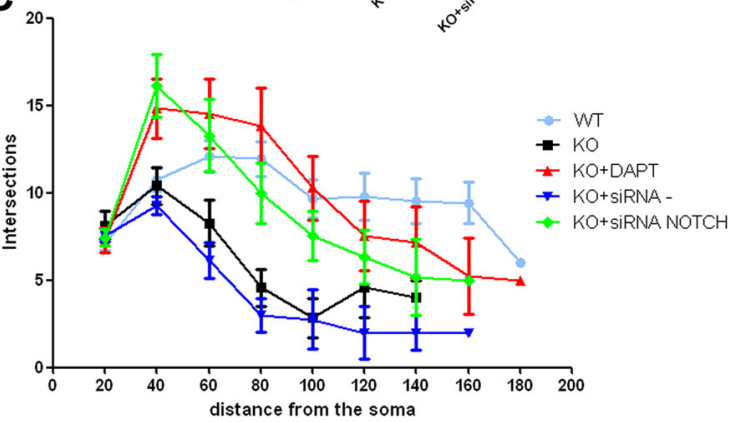

Figure 5. Notch1 blockade restores neurite branching and varicosities in $\mathrm{p}^{-1-}$ cortical neurons. $A$, Representative confocal images of cortical cells stained with anti- $\beta$ III-Tubulin antibody (red), DAPI (blue), and anti-Notch1 antibody (green). K0 cortical neurons were exposed to the $\gamma$-secretase inhibitor DAPT, the siRNA NOTCH, or the scrambled siRNA probe (siRNA -) for $72 \mathrm{~h}$. Scale bar, $20 \mu \mathrm{m}$. $B$, Quantification of varicosity density in WT and K0 neurons after different treatments, as indicated. Varicosity density was measured counting number of varicosities in $100 \mu \mathrm{m}$ neurite length. Data are expressed as mean \pm SEM. ${ }^{*} p<0.001$ versus KO. C, Graphic representation of branching quantification performed using the Sholl profile analysis in WT and KO neurons after different treatments, as indicated. Data are expressed as mean \pm SEM.

phological abnormalities in $\mathrm{p} 50^{-/-}$cortical neurons are dependent on Notch pathway hyperactivation.

No morphological changes were observed in wt cortical neurons treated with either DAPT or siRNA Notch (data not shown).

\section{p50 NF- $\kappa \mathrm{B}$ subunit is directly involved in Notch pathway modulation}

NF- $\kappa \mathrm{B}$ signaling was acutely manipulated using SN-50 peptide (Lin et al., 1995). Treatment of wt cells with $10 \mu \mathrm{M} \mathrm{SN}-50$ for $48 \mathrm{~h}$ 
resulted in increased Jagged $1(+191 \%)$ and Notch1 $(+66 \%)$ mRNA levels, compared with vehicle- or mutated SN-50 (SN-50 mut)-treated cells (Fig. 6A). Branching studies by Sholl analysis revealed that SN-50 significantly reduced neurite length and complexity in wild-type neurons (Fig. 6B). Also, varicosities were reduced after SN-50 treatment: varicosity densities were $7.53 \pm$ $0.41,8.24 \pm 0.42$, and $3.00 \pm 0.26$ in vehicle-, SN-50 mut-, and SN-50-treated cortical cells, respectively.

We evaluated the role of p50 in regulating Jagged 1 expression in mouse neuronal cortical cells using the ChIP approach. In line with previous data (Johnston et al., 2009), we found that vehicle-treated cells yielded a strong signal for p50 and only a weak p65 signal on the Jagged 1 promoter (Fig. 6C). In sharp contrast to control cells, this ratio is reversed in TNF-treated cells, in which we found a weak p50 signal and a strong p65 signal, correlating with the higher transcriptional activity of the Jagged 1 gene in TNF-treated cells (Fig. 6D). A similar pattern of results was obtained in cells treated with SN-50. Together, the TNF- $\alpha$ and $\mathrm{SN}-50$ results suggest that, in resting cells, the NF- $\kappa \mathrm{B}$ site is likely occupied mostly by $\mathrm{p} 50$ homodimers, whereas in TNF- $\alpha$ - or SN-50-treated cells there is a shift toward p65-containing complexes, and this event is associated with enhanced Jagged 1 transcription. As it is not entirely clear what the consequences of deletion of p50 throughout embryogenesis may have for NF- $\kappa \mathrm{B}$ signaling in cortical neurons, we evaluated $\mathrm{p} 65$ protein levels in $\mathrm{p} 50^{-1-}$ and wt cortical neurons. We found that $\mathrm{p} 50^{-/-}$ neurons expressed significantly higher protein level (+152\%) compared with wt.

\section{Localization of Notch1- and Jagged1-immunoreactive} neurons in $\mathrm{p}^{50^{-/-}} \mathrm{P} 1$ mice cortex

Finally, we investigated whether Notch1 hyperactivation observed in

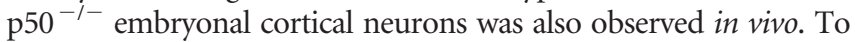

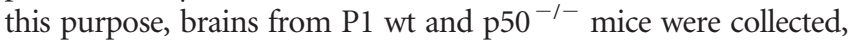
fixed, cut in 15- $\mu \mathrm{m}$-thick coronal sections, and immunostained for Notch1 and Jagged1 proteins. In P1 wt mice, Notch1 immunoreactivity was detected in the basal zone of the motor/somatosensory cortex and gradually decreased in intensity toward the upper cortical layers, as shown in the representative images in Figure 7A, top panel. Conversely, in $\mathrm{p}^{-1-}$ brain sections, Notch1-immunoreactive cells were detected throughout all cortical layers (Fig. 7A, bottom panel). A similar expression pattern was also observed for Jagged 1 immunofluorescence in $\mathrm{p}^{-1-}$ compared with wt brains: Jagged1positive cells were mainly localized in the basal cortical layer in P1 wt brain sections, whereas in $\mathrm{p} 50^{-/-}$mice, Jagged1-positive cells were spread throughout the upper layers (Fig. 7B).

\section{Discussion}

In the CNS, activation of NF- $\kappa \mathrm{B}$ has been reported to be involved in neuronal cell differentiation and survival (Maggirwar et al.,

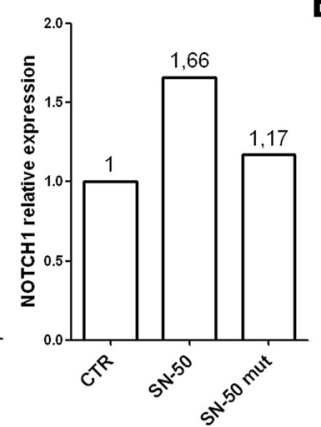

36B4
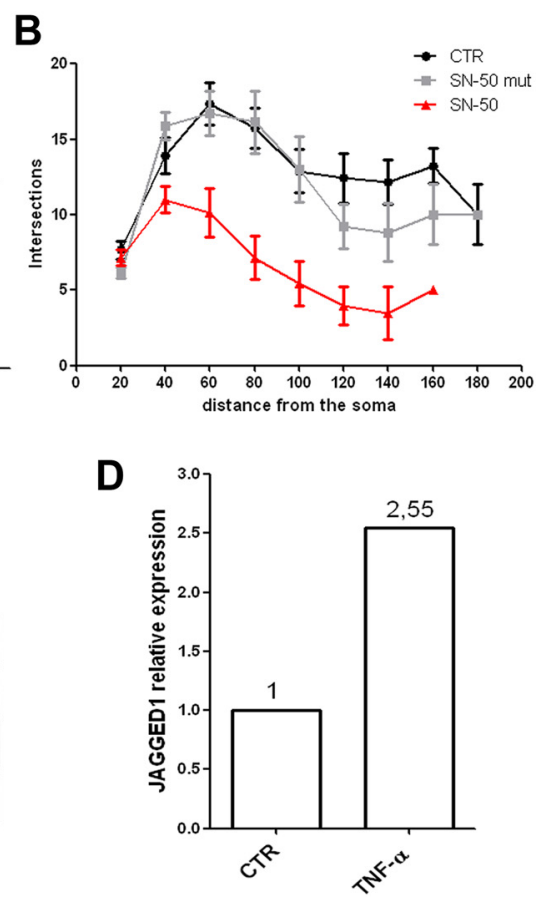

Figure 6. Acute NF- $\kappa B$ signaling manipulation induces Jagged1 upregulation and morphological changes. A, Q-RT-PCR was performed on cDNA from WT cortical cells after $10 \mu \mathrm{m} \mathrm{SN-50}$ or SN-50 mut treatment at $48 \mathrm{~h}$. Data are presented as fold change of An SN-50 mutant peptide (SN-50 mut) was used to validate SN-50 specificity. B, Graphic representation of branching quantifica列 Additiona controts a sample representing linear amplification of the total input chromatin (Input) was included as contro WT cortical cells after $24 \mathrm{~h}$ of TNF- $\alpha$ treatment $(10 \mathrm{ng} / \mathrm{ml})$. Data are presented as fold change of target gene expression normalized upregulation of Jagged1 mRNA expression compared with the vehicle-treated cells (CTR).

1998; Kovács et al., 2004), inflammatory response (Li and Verma, 2002), neurogenesis (Denis-Donini et al., 2005, 2008), proliferation, and apoptosis (Grilli and Memo, 1999; Kucharczak et al., 2003). More recently, emerging data demonstrated its crucial involvement in regulating the growth and complexity of neuronal arborizations (Gutierrez et al., 2005; Gavaldà et al., 2009; Russo et al., 2009; Gutierrez and Davies, 2011) and in synaptic plasticity and memory in the adult brain (Albensi and Mattson, 2000; Kaltschmidt et al., 2006). Nevertheless, the mechanisms by which NF- $\kappa \mathrm{B}$ may exert such a role is largely unknown.

$\mathrm{p} 50^{-1-}$ mice were chosen as an experimental model to investigate the contribution of NF- $\kappa \mathrm{B}$ in regulating cortical cell morphology and to identify the intracellular pathway(s) involved in such effects. p50 ${ }^{-1-}$ mice have been extensively used in an attempt to clarify the role of NF- $\kappa \mathrm{B}$ in developing, adult, and aged nervous system. Indeed, this mouse model has been shown to display accelerated aging (Lu et al., 2006) and a dramatic impairment in hippocampal neurogenesis linked to a selective cognitive deficit in hippocampal-dependent spatial short-term memory (Denis-Donini et al., 2008). Mice lacking p50 subunit also display an increased exploratory activity and reduced anxiety behavior (Kassed and Herkenham, 2004).

In the present study, measuring parameters commonly used to define the stage of neuron differentiation during brain devel- 
A
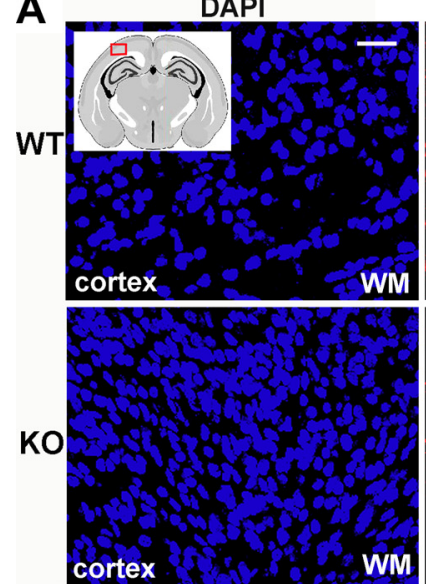

B
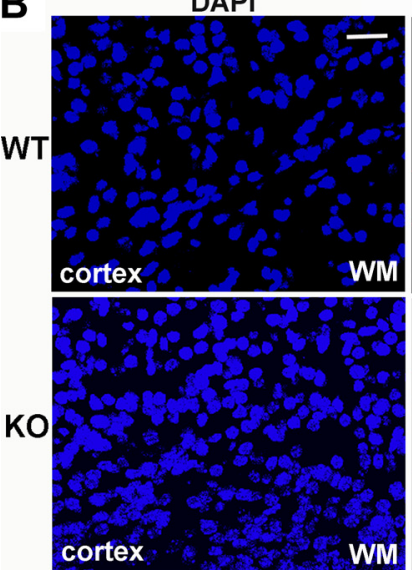

WM
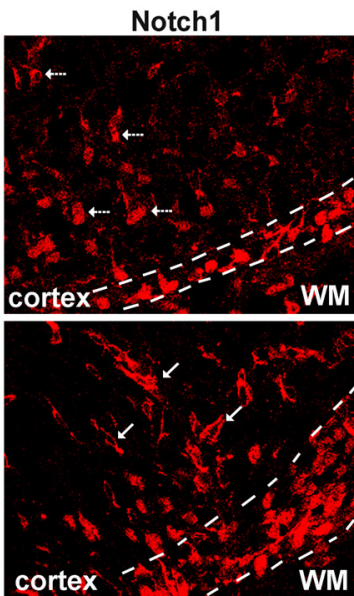

Jagged1

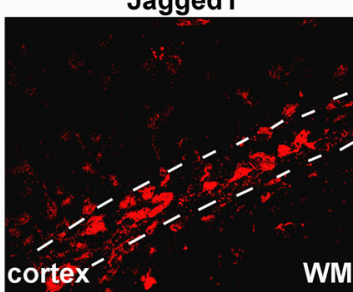

WM

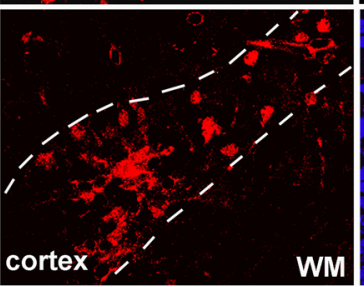

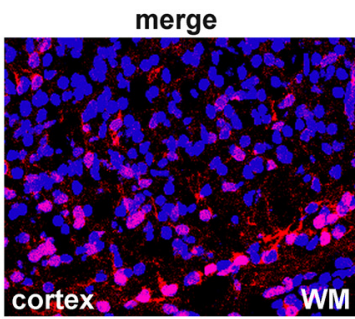

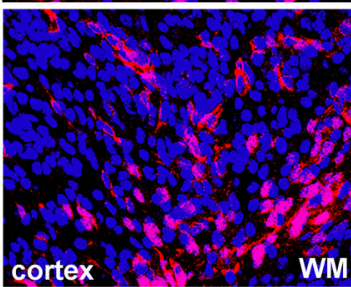

merge
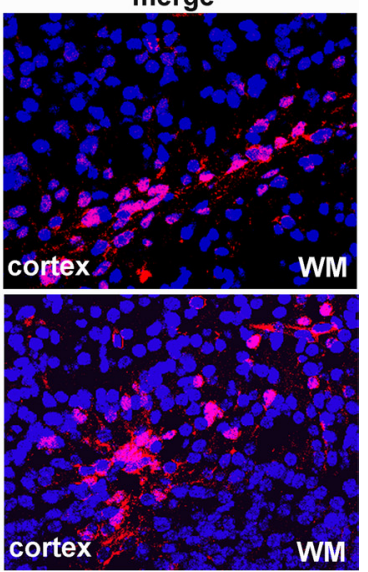

Figure 7. In vivo increase of Notch1- and Jagged1-positive neurons in $\mathrm{p}^{-1-} \mathrm{O}^{-1-}$ mouse cortex. Representative confocal images of cerebral cortex from P1 WT and K0 mouse brain sections stained with anti-Notch1 (red) or anti-Jagged1 (red) antibody and DAPI (blue). Inset, Scheme of a P1 mouse brain section, with the indication (red mark) of the region of interest. $A$, Top panel, Representative images of WT cortex with Notch1-positive neurons localized mainly in the basal layer and few Notch1-stained cells in the upper cortical layers (arrows). Bottom panel, Representative images of KO mouse cortex with Notch1-expressing neurons both in the basal cortical layer and in the whole cortical stratifications (arrows). $\boldsymbol{B}$, Top panel, Representative images of WT cortex with Jagged1-positive neurons localized in the basal layer. Bottom panel, Representative images of $\mathrm{K} 0$ mouse cortex with Jagged1expressing neurons that exceed the basal layer and spread through other cortical layers. WM, White matter. Scale bar, $20 \mu \mathrm{m}$.

opment, we unraveled marked morphological and biochemical differences between wt and $\mathrm{p}^{-1-}$ cortical cells in culture. We demonstrated that neuritic branching and varicosity density were significantly reduced in $\mathrm{p}^{-1-}$ neurons. Our results on the $\mathrm{NF}-\kappa \mathrm{B}$ role in neurite growth regulation are in line with data from Gutierrez et al. $(2007,2008)$ in nodose and sensory neurons. These authors reported that different protein subunits contribute to NF- $\kappa \mathrm{B}$-mediated effect on neurites, and, in particular, they described a remarkable inhibitory effect of SN-50, a peptide that interferes with p50 nuclear translocation, on neuritic branching. We now further extend these observations, demonstrating the role of NF- $\kappa \mathrm{B}$ on neuronal branching and differentiation also in cortical neurons.

We were interested in investigating the mechanism by which NF- $\kappa \mathrm{B}$ is able to act on neuritic morphology. Using primary murine hippocampal cultures, Salama-Cohen et al. (2005) provided evidence that Notch and NF- $\kappa \mathrm{B}$ signaling pathways converge at the level of Hes1/5 to regulate dendritic growth. In previous studies, we characterized the effects of Notch1 in remodeling neuronal processes, analyzing its effect on cytoskeleton, varicosities, and neurotransmitter release (Ferrari-Toninelli et al., 2008, 2009). Surprisingly, the reduced varicosity density and neurite ramifications observed in $\mathrm{p} 50^{-1-}$ neurons strongly resembled the morphology seen in cortical neurons or differentiated SH-SY5Y cells after Notch1 receptor stimulation by its ligand Jagged1. Since the relevance of Notch signaling in development and synaptic transmission, it appeared reasonable to analyze the Notch1 pathway in $\mathrm{p}^{-1-}$ cortical cells.

Notch1 is well known for its involvement in important cellular processes like neurogenesis (Artavanis-Tsakonas et al., 1999), cell fate determination (Louvi and Artavanis-Tsakonas, 2006), and neural stem cell maintenance in the adult brain (Imayoshi et al., 2010). As recently reviewed by Ables et al. (2011), several studies suggested that Notch signaling may serve important functions in the regulation of neurite outgrowth and plasticity. Both Šestan et al. (1999) and Berezovska et al. (1999) found that Notch1 activation inhibits neurite outgrowth or causes their retraction, whereas Notch1 signaling inhibition promotes neurite extension. Redmond et al. (2000) demonstrated that NICD translocates to the nucleus during neuronal differentiation where it regulates the expression of genes whose products influence dendritic morphology. Here, we collected evidence indicating that the Notch signaling is hyperactivated in $\mathrm{p}^{-1-}$ cortical neurons. Remarkably, Jagged1 transcript was increased by 17 fold in $\mathrm{p}^{-1-}$ cells compared with wt cells, suggesting that it might be directly affected by the lack of p50 subunit. We manipulated acutely NF- $\kappa \mathrm{B}$ signaling in wt neurons. Specifically, wt cortical cultures were treated with $\mathrm{SN}-50$ and resulted in a significant increase of both Jagged 1 and Notch 1 mRNA levels. SN-50-treated cells also displayed a significant reduction in neurite branching and varicosity density. Using the ChIP approach, we found that, in resting cells, the NF- $\kappa \mathrm{B}$ site within Jagged 1 promoter is occupied mostly by p50 homodimers, whereas in TNF- $\alpha$ or SN-50 treated cells there is a shift toward p65-containing complexes, which correlates with enhanced Jagged 1 transcription. This is in line with the data by Johnston et al. (2009) demonstrating that, in human endothelial cells, Jagged 1 transcription is repressed by $\mathrm{p} 50$ homodimers. Our data suggest that, in wt culture system, p50-containing dimers may act as repressor of Jagged 1 gene expression. A vast array of information is available in the literature (Zhong et al., 2002; Driessler et al., 2004; Grundström et al., 2004) describing the specific role of p50 homodimers as transcriptional repressors within the family.

We propose Jagged 1 as one of the major players in Notch pathway hyperactivation. Its interaction with the receptor may indeed result in massive translocation of NICD to the nucleus and thereby in increased transcription of the Notch target gene HES1. HES1 is a Notch1-responsive bHLH family member implicated in controlling the maintenance of undifferentiated cells and timing of cell differentiation (Akimoto et al., 2010). Since 
different Notch receptors and ligands have been described, we cannot exclude the possible involvement of other members of the Notch pathway in this effect. However, in our in vitro model the increased NICD nuclear levels and HES1 expression unequivocally testify for a Notch pathway hyperactivity.

We were then interested in evaluating the possible functional link between morphological features and Notch pathway activity. To test this hypothesis, the Notch pathway was inhibited and morphology of cortical neurons was studied. Two different approaches were undertaken: a pharmacological treatment with the $\gamma$-secretase inhibitor DAPT, and Notch 1 gene silencing. DAPT is a well known Notch1 pharmacological inhibitor (Sastre et al., 2001; Geling et al., 2002; Crawford and Roelink, 2007), preventing Notch1 intracellular cleavage, which is required for NICD nuclear translocation and signaling activation. DAPT exerts a strong inhibitory effect on Notch1 activation, but it has poor selectivity, because of the high number of $\gamma$-secretase substrates (Lleó, 2008). siRNA NOTCH is a Notch1 gene interference tool that specifically downregulates Notch1 transcription. Both experimental treatments resulted effective in inhibiting the Notch1 signaling. Interestingly, treatment of $\mathrm{p} 50^{-1-}$ cortical cells with either DAPT or siRNA NOTCH was able to recover varicosities loss and increase neurite branching. These findings indicated that the morphological alterations present in cortical neurons lacking the NF- $\kappa \mathrm{B}$ p50 subunit are dynamic and reversible. More importantly, these results also suggested that Notch hyperactivation mediated the morphological features observed in the absence of the NF- $\kappa \mathrm{B}$ p50 subunit.

Notch effects on neurite plasticity might be relevant to the generation of highly refined neuronal circuits (Luo and O'Leary, 2005). Axonal and dendrite architecture is indeed responsible for polarization and migration of neuronal cells and crucial for correct lamination during cortex development. The analysis of p50 $0^{-1-}$ and wt mouse brains, at postnatal day 1 , revealed an altered distribution of Notch1- and Jagged1-positive cells in the cortex. In control brains, Notch1- and Jagged 1-positive cells were uniformly localized in the basal layer of the cortex, with few cells in the upper layers. Conversely, $\mathrm{p} 50^{-1-}$ mice showed an increased number of both Notch1- and Jagged1-expressing cells, with a distribution not only in basal but also in upper layers. In this regard, it should be noted that the Notch pathway has been found to be implicated in cortical neuron migration and in cortical layering, mainly through the cross talk with the Reelin signaling pathway (Gaiano, 2008; Hashimoto-Torii et al., 2008). Although young and adult $\mathrm{p} 50^{-1-}$ mouse brains do not show overt structural alterations, our data suggest that future studies will be needed to address the possibility that in absence of p50 very subtle defects may occur in neuronal networks and in particular in cortex and that they may potentially contribute to behavioral alterations.

In summary, we found that lack of p50, either induced acutely by inhibiting p50 nuclear translocation in wt cortical cells or genetically in $\mathrm{p}^{-1-}$ mice, results in cortical neurons characterized by reduced neurite branching, loss of varicosities, and Notch1 signaling hyperactivation. The neuronal morphological effects caused by lack of p50 were reverted by inhibition of Notch signaling.

A direct link between Notch and NF- $\kappa$ B pathways is also supported by ChIP data showing that activation of Jagged1 gene transcription is associated with removal of p50 homodimers bound to the NF- $\kappa \mathrm{B}$-responsive element in the Jagged1 promoter region.

These data demonstrate that a cross talk between NF- $\kappa \mathrm{B}$ and Notch1 signaling in primary neuronal cortical cells does exist.
Our data also support a role for this interaction in the regulation of cortical neuron remodeling.

\section{References}

Ables JL, Breunig JJ, Eisch AJ, Rakic P (2011) Not(ch) just development: Notch signalling in the adult brain. Nat Rev Neurosci 12:269-283.

Akimoto M, Kameda Y, Arai Y, Miura M, Nishimaki T, Takeda A, Uchinuma E (2010) HES1 is required for the development of craniofacial structures derived from ectomesenchymal neural crest cells. J Craniofac Surg 21:1443-1449.

Albensi BC, Mattson MP (2000) Evidence for the involvement of TNF and NF- $\kappa B$ in hippocampal synaptic plasticity. Synapse 35:151-159.

Ang HL, Tergaonkar V (2007) Notch and NF- $\kappa$ B signaling pathways: do they collaborate in normal vertebrate brain development and function? Bioessays 29:1039-1047.

Artavanis-Tsakonas S, Delidakis C, Fehon R, Hartley D, Herndon D, Johansen K, Markopoulou K, Preiss A, Rebay I, Scottgale N (1990) Notch and the molecular genetics of neuroblast segregation in Drosophila. Mol Reprod Rev 27:23-27.

Artavanis-Tsakonas S, Rand MD, Lake RJ (1999) Notch signaling: cell fate control and signal integration in development. Science 284:770-776.

Bash J, Zong WX, Banga S, Rivera A, Ballard DW, Ron Y, Gélinas C (1999) $\mathrm{Rel} / \mathrm{NF}-\kappa \mathrm{B}$ can trigger the Notch signaling pathway by inducing the expression of Jagged1, a ligand for Notch receptors. EMBO J 18:2803-2811.

Berezovska O, McLean P, Knowles R, Frosh M, Lu FM, Lux SE, Hyman BT (1999) Notch1 inhibits neurite outgrowth in postmitotic primary neurons. Neuroscience 93:433-439.

Cao Q, Li P, Lu J, Dheen ST, Kaur C, Ling EA (2010) Nuclear factor $-\kappa B / p 65$ responds to changes in the Notch signaling pathway in murine BV-2 cells and in amoeboid microglia in postnatal rats treated with the $\gamma$-secretase complex blocker DAPT. J Neurosci Res 88:2701-2714.

Crawford TQ, Roelink H (2007) The Notch response inhibitor DAPT enhances neuronal differentiation in embryonic stem cell-derived embryoid bodies independently of sonic hedgehog signaling. Dev Dyn 236: 886-892.

de la Pompa JL, Wakeham A, Correia KM, Samper E, Brown S, Aguilera RJ, Nakano T, Honjo T, Mak TW, Rossant J, Conlon RA (1997) Conservation of the Notch signaling pathway in mammalian neurogenesis. Development 124:1139-1148.

Denis-Donini S, Caprini A, Frassoni C, Grilli M (2005) Members of the NF- $\kappa \mathrm{B}$ family expressed in zones of active neurogenesis in the post-natal and adult mouse brain. Brain Res Dev Brain Res 154:81-89.

Denis-Donini S, Dellarole A, Crociara P, Francese MT, Bortolotto V, Quadrato G, Canonico PL, Orsetti M, Ghi P, Memo M, Bonini SA, FerrariToninelli G, Grilli M (2008) Impaired adult neurogenesis associated with short-term memory defects in NF- $\kappa$ B p50-deficient mice. J Neurosci 28:3911-3919.

De Paola V, Arber S, Caroni P (2003) AMPA receptors regulate dynamic equilibrium of presynaptic terminals in mature hippocampal networks. Nat Neurosci 6:491-500.

Driessler F, Venstrom K, Sabat R, Asadullah K, Schottelius AJ (2004) Molecular mechanisms of interleukin-10-mediated inhibition of NF- $\kappa \mathrm{B}$ activity: a role for p50. Clin Exp Immunol 135:64-73.

Espinosa L, Inglés-Esteve J, Robert-Moreno A, Bigas A (2003) I $\kappa \mathrm{B} \alpha$ and p65 regulate the cytoplasmic shuttling of nuclear corepressors: cross-talk between Notch and NFкB pathways. Mol Biol Cell 14:491-502.

Ferrari-Toninelli G, Bonini SA, Bettinsoli P, Uberti D, Memo M (2008) Microtubule stabilizing effect on Notch activation in primary cortical neurons. Neuroscience 154:946-952.

Ferrari-Toninelli G, Bonini SA, Uberti D, Napolitano F, Stante M, Santoro F, Minopoli G, Zambrano N, Russo T, Memo M (2009) Notch activation induces neurite remodeling and functional modifications in SH-SY5Y neuronal cells. Dev Neurobiol 69:378-391.

Gaiano N (2008) Strange bedfellows: Reelin and Notch signaling interact to regulate cell migration in the developing neocortex. Neuron 60:189-191.

Gavaldà N, Gutierrez H, Davies AM (2009) Developmental switch in NF- $\kappa$ B signaling required for neurite growth. Development 136:3405-3412.

Geling A, Steiner H, Willem M, Bally-Cuif L, Haass C (2002) A gammasecretase inhibitor blocks Notch signaling in vivo and causes a severe neurogenic phenotype in zebrafish. EMBO Rep 3:688-694.

Grilli M, Memo M (1999) Nuclear factor- $\kappa$ B/Rel proteins. Biochem Pharmacol 57:1-7. 
Grundström S, Anderson P, Scheipers P, Sundstedt A (2004) Bcl-3 and $\mathrm{NF} \kappa \mathrm{B}$ p50-p50 homodimers act as transcriptional repressors in tolerant CD4+ T cells. J Biol Chem 279:8460-8468.

Gutierrez H, Davies AM (2007) A fast and accurate procedure for deriving the Sholl profile in a quantitative studies of neuronal morphology. J Neurosci Methods 163:24-30.

Gutierrez H, Davies AM (2011) Regulation of neural process growth, elaboration and structural plasticity by NF- $\kappa$ B. Trends Neurosci 34:316-325.

Gutierrez H, Hale VA, Dolcet X, Davies A (2005) NF- $\kappa$ B signaling regulates the growth of neural processes in the developing PNS and CNS. Development 132:1713-1726.

Gutierrez H, O’Keeffe GW, Gavaldà N, Gallagher D, Davies AM (2008) Nuclear factor $\kappa \mathrm{B}$ signaling either stimulates or inhibits neurite growth depending on the phosphorylation status of p65/RelA. J Neurosci 28:8246-8256.

Hashimoto-Torii K, Torii M, Sarkisian MR, Bartley CM, Shen J, Radtke F, Gridley T, Šestan N, Rakic P (2008) Interaction between Reelin and Notch signaling regulates neuronal migration in the cerebral cortex. Neuron 60:273-284.

Imayoshi I, Sakamoto M, Yamaguchi M, Mori K, Kageyama R (2010) Essential roles of Notch signaling in maintenance of neural stem cells in developing and adult brains. J Neurosci 30:3489-3498.

Iso T, Kedes L, Hamamori Y (2003) HES and HERP families: multiple effectors of the Notch signaling pathway. J Cell Physiol 194:237-255.

Johnston DA, Dong B, Hughes CC (2009) TNF induction of jagged-1 in endothelial cells is NF- $\kappa$ B-dependent. Gene 435:36-44.

Kaltschmidt B, Kaltschmidt C (2009) NF- $\kappa$ B in the nervous system. Cold Spring Harb Perspect Biol 1:a001271.

Kaltschmidt B, Ndiaye D, Korte M, Pothion S, Arbibe L, Prüllage M, Pfeiffer J, Lindecke A, Staiger V, Israël A, Kaltschmidt C, Mémet S (2006) NF- $\kappa$ B regulates spatial memory formation and synaptic plasticity through protein kinase A/CREB signaling. Mol Cell Biol 26:2936-2946.

Kanungo J, Zheng YL, Amin ND, Pant HC (2008) The Notch signaling inhibitor DAPT down-regulates cdk5 activity and modulates the distribution of neuronal cytoskeletal proteins. J Neurochem 106:2236-2248.

Kassed CA, Herkenham M (2004) NF- $\kappa$ B p50-deficient mice show reduced anxiety-like behaviours in test of exploratory drive and anxiety. Behav Brain Res 154:577-584.

Kovács AD, Chakraborty-Sett S, Ramirez SH, Sniderhan LF, Williamson AL, Maggirwar SB (2004) Mechanism of NF- $\kappa$ B inactivation induced by survival signal withdrawal in cerebellar granule neurons. Eur J Neurosci 20:345-352.

Kucharczak J, Simmons MJ, Fan Y, Gélinas C (2003) To be, or not to be: $\mathrm{NF}-\kappa \mathrm{B}$ is the answer. Role of Rel/NF- $\kappa \mathrm{B}$ in the regulation of apoptosis. Oncogene 22:8961-8982.

Lanni C, Nardinocchi L, Puca R, Stanga S, Uberti D, Memo M, Govoni S, D’Orazi G, Racchi M (2010) Homeodomain interacting protein kinase 2: a target for Alzheimer's beta amyloid leading to misfolded p53 and inappropriate cell survival. PLoS One 5:e10171.

Li Q, Verma IM (2002) NF- $\kappa$ B regulation in the immune system. Nat Rev Immunol 2:725-734.

Lin YZ, Yao SY, Veach RA, Torgerson TR, Hawiger J (1995) Inhibition of nuclear translocation of transcription factor NF- $\kappa$ B by a synthetic peptide containing a cell membrane-permeable motif and nuclear localization sequence. J Biol Chem 270:14255-14258.

Lleó A (2008) Activity of gamma-secretase on substrates other than APP. Curr Top Med Chem 8:9-16.

Louvi A, Artavanis-Tsakonas S (2006) Notch signaling in vertebrate neural development. Nat Rev Neurosci 7:93-102.

Lu ZY, Yu SP, Wei JF, Wei L (2006) Age-related neural degeneration in nuclear-factor $\kappa \mathrm{B}$ p50 knockout mice. Neuroscience 139:965-978.

Luo L, O'Leary DD (2005) Axon retraction and degeneration in development and disease. Annu Rev Neurosci 28:127-156.

Maggirwar SB, Sarmiere PD, Dewhurst S, Freeman RS (1998) Nerve growth factor-dependent activation of NF- $\kappa$ B contributes to survival of sympathetic neurons. J Neurosci 18:10356-10365.

Mattson MP (2005) NF- $\kappa$ B in the survival and plasticity of neurons. Neurochem Res 30:883-893.

Mattson MP, Meffert MK (2006) Roles for NF- $\kappa$ B in nerve cell survival, plasticity, and disease. Cell Death Differ 13:852-860.

Nickoloff BJ, Qin JZ, Chaturvedi V, Denning MF, Bonish B, Miele L (2002) Jagged-1 mediated activation of notch signaling induces complete maturation of human keraticocytes through NF- $\kappa \mathrm{B}$ and PPAR $\gamma$. Cell Death Differ 9:842-855.

Nikonenko I, Jourdain P, Muller D (2003) Presynaptic remodeling contributes to activity-dependent synaptogenesis. J Neurosci 23:8498-8505.

Osipo C, Golde TE, Osborne BA, Miele LA (2008) Off the beaten pathway: the complex cross talk between Notch and NF- $\kappa$ B. Lab Invest 88:11-17.

Redmond L, Oh SR, Hicks C, Weinmaster G, Ghosh A (2000) Nuclear Notch1 signaling and the regulation of dendritic development. Nat Neurosci 3:30-40.

Rozen S, Skaletsky H (2000) Primer3 on the WWW for general users and for biologist programmers. Methods Mol Biol 132:365-386.

Russo SJ, Wilkinson MB, Mazei-Robison MS, Dietz DM, Maze I, Krishnan V, Renthal W, Graham A, Birnbaum SG, Green TA, Robison B, Lesselyong A, Perrotti LI, Bolaños CA, Kumar A, Clark MS, Neumaier JF, Neve RL, Bhakar AL, Barker PA, et al. (2009) Nuclear factor $\kappa$ B signaling regulates neuronal morphology and cocaine reward. J Neurosci 29:3529-3537.

Salama-Cohen P, Arévalo MA, Meier J, Grantyn R, Rodríguez-Tébar A (2005) NGF controls dendrite development in hippocampal neurons by binding to $75^{\mathrm{NTR}}$ and modulating the cellular targets of Notch. Mol Biol Cell 16:339-347.

Sastre M, Steiner H, Fuchs K, Capell A, Multhaup G, Condron MM, Teplow DB, Haass C (2001) Presenilin-dependent gamma-secretase processing of beta-amyloid precursor protein at a site corresponding to the S3 cleavage of Notch. EMBO Rep 2:835-841.

Šestan N, Artavanis-Tsakonas S, Rakic P (1999) Contact-dependent inhibition of cortical neurite growth mediated by Notch signaling. Science 286:741-746.

Sha WC, Liou HC, Tuomanen EI, Baltimore D (1995) Targeted disruption of the p50 subunit of NF-kappaB leads to multifocal defects in immune responses. Cell 80:321-330.

Shin HM, Minter LM, Cho OH, Gottipati S, Fauq AH, Golde TE, Sonenshein GE, Osborne BA (2006) Notch 1 augments NF- $\kappa$ B activity by facilitating its nuclear retention. EMBO J 25:129-138.

Sholl DA (1953) Dendritic organization in the neurons of the visual and motor cortices of the cat. J Anat 87:387-406.

Udo H, Jin I, Kim JH, Li HL, Youn T, Hawkins RD, Kandel ER, Bailey CH (2005) Serotonin-induced regulation of the actin network for learningrelated synaptic growth requires Cdc42, N-WASP, and PAK in Aplysia sensory neurons. Neuron 45:887-901.

Umeda T, Ebihara T, Okabe S (2005) Simultaneous observation of stably associated presynaptic varicosities and postsynaptic spines: morphological alterations of CA3-CA1 synapses in hippocampal slice cultures. Mol Cell Neurosci 28:264-274.

van Spronsen M, Hoogenraad CC (2010) Synapse pathology in psychiatric and neurologic disease. Curr Neurol Neurosci Rep 10:207-214.

Wang J, Shelly L, Miele L, Boykins R, Norcross MA, Guan E (2001) Human Notch-1 inhibits NF- $\kappa$ B activity in the nucleus through a direct interaction involving a novel domain. J Immunol 167:289-295.

Wang Y, Chan SL, Miele L, Yao PJ, Mackes J, Ingram DK, Mattson MP, Furukawa K (2004) Involvement of Notch signaling in hippocampal synaptic plasticity. Proc Natl Acad Sci U S A 101:9458-9462.

Williams R, Lendahl U, Lardelli M (1995) Complementary and combinatorial patterns of Notch gene family expression during early mouse development. Mech Dev 53:357-368.

Zhong H, May MJ, Jimi E, Ghosh S (2002) The phosphorylation status of nuclear NF- $\kappa \mathrm{B}$ determines its association with $\mathrm{CBP} / \mathrm{p} 300$ or HDAC-1. Mol Cell 9:625-636. 